\title{
Educational and training program of THz Science and Technology at Rensselaer
}

G.-C. Wang, T.-M. Lu, M. Shur, S. Kalyanaraman, X.-C. Zhang

G.-C. Wang, T.-M. Lu, M. Shur, S. Kalyanaraman, X.-C. Zhang, "Educational and training program of THz Science and Technology at Rensselaer," Proc. SPIE 9663, Eighth International Topical Meeting on Education and Training in Optics and Photonics, 966300 (6 October 2003); doi: 10.1117/12.2207353

SPIE Event: Eighth International Topical Meeting on Education and Training in Optics and Photonics, 2003, Tucson, Arizona, United States 


\title{
Educational and Training program of THz Science and Technology at Rensselaer
}

\author{
G.-C. Wang, T.-M. Lu, M. Shur, S. Kalyanaraman, and X.-C. Zhang \\ Rensselaer Polytechnic Institute, Troy, NY 12180 USA \\ P/F (518) 276 3079/3292; email: zhangxc@rpi.edu; web: http://www.rpi.edu/terahertz/
}

\begin{abstract}
Rensselaer is establishing an educational program, THz Science and Technology, with an interdisciplinary faculty team from Departments of Physics, Biology, and Electrical, Computer, and Systems Engineering. Doctoral level students are trained in $\mathrm{THz}$ electronics, $\mathrm{THz}$ spectroscopy, THz imaging, and $\mathrm{THz}$ data transfer and networking. We present examples of focus research and studio based education.

(C2003 Optical Society of America

OCIS codes: (320.0320) Ultrafast optics; (250.0250) Optoelectronics ; (110.0110) Imaging systems
\end{abstract}

\section{Introduction}

In July 2002, Rensselaer Polytechnic Institute established the Center for Terahertz Research. The Center is initiating an integrated education and training program on $\mathrm{THz}$ Science and Technology with a team-based, highly interactive, and hands-on studio-based approach.

Under the Rensselaer Plan, adopted in 2000, Rensselaer has chosen to focus investment and resources in information science and biotechnology. Systems or devices that operate in the $\mathrm{THz}$ regime are expected to revolutionize both. Proposed novel devices, such as $\mathrm{THz}$ plasma wave electronics, molecular spintronics, $\mathrm{THz}$ modulators, and photonic bandgap switches will transform future computing, networking, and communication systems. At the same time, THz systems will provide unique opportunities for biomedical imaging, including 3D tomography.

Rensselaer's $\mathrm{THz}$ research program is interdisciplinary, spanning physics, electrical engineering, computer science, biology, and other departments. The research cuts across major centers on campus, including the Center for $\mathrm{THz}$ Research, the Center for Broadband Data Transport, the Center for Advanced Interconnect Systems Technologies, and the Nanotechnology Center. Rensselaer is strongly committed to THz research and has provided more than 5,000 square feet of laboratory space for centralized $\mathrm{THz}$ research and education, equipped with the help of a $\$ 1$ million grant from the Keck Foundation.

Rensselaer plans to offer specialized $\mathrm{THz}$ courses, for example, $\mathrm{THz}$ wave science and technology in imaging/spectroscopy and $\mathrm{THz}$ device technology, which will be created and taught in an interactive studio setting by interdisciplinary faculty teams. Besides these studio courses, there will be industrial and international internship opportunities. There will also be special programs in leadership, ethics, life skills, global citizenship, and outreach.

The strength of Rensselaer's $\mathrm{THz}$ program offers an exciting opportunity to create a new model for interdisciplinary, doctoral-level graduate education that offers students both broad exposure and in depth training.

\section{Core Physicists in the Center for THz Research and its Near Term Research Focus}

$\mathrm{THz}$ research at Rensselaer Polytechnic Institute started in the Department of Physics, Applied Physics and Astronomy. Since the first hiring of a THz faculty member (Prof. X.-C. Zhang) from Columbia University in 1992, the department has recruited two more faculty members (Prof. R. Kersting and Prof. I. Wilke) in THz field. In the Rensselaer Performance Plan, the School of Science will hire three additional faculty members in the next four years. These faculty members create the critical mass for $\mathrm{THz}$ research at Rensselaer.

During the last several years, scientists and engineers from more than 75 universities, companies, and clinics have visited Rensselaer. Rensselaer's THz Group has helped scientists from 18 countries learn to use THz sensors in their respective fields. Based on research and education needs, Rensselaer President Dr. Shirley Jackson approved the formation of the $\mathrm{THz}$ Center. The Center for $\mathrm{THz}$ Research at Rensselaer consisting of four core faculty members was officially announced by the School of Science Dean Joseph Flaherty on July 3, 2002. Two days later, the Institute received word that the W. M. Keck Foundation has awarded Rensselaer \$1 million to establish the W. M. Keck Laboratory for Terahertz Science within the new research center. The promise of terahertz wave radiation, known as "T-rays", is being realized through ongoing research at the THz Center's state-of-the-art laboratories: Dr.

Eighth International Topical Meeting on Education and Training in Optics and Photonics,

edited by Barry L. Shoop, Grover Swartzlander Jr., Proc. of SPIE Vol. 9663, 966300

(c) 2003 SPIE, OSA, ICO · doi: 10.1117/12.2207353 
Xi-Cheng Zhang's THz Imaging Lab, Dr. Michael Shur's THz Electronics Lab, Dr. Roland Kersting's THz Quantum Optics Lab, and Dr. Ingrid Wilke's THz Spectroscopic Lab.

The Center's Focused Topics: As a focused research team, the Center will concentrate on five topics in the near future:

THz Spectroscopy and Imaging: A team works on the study of protein interactions on surfaces, and protein/protein interactions using $\mathrm{THz}$ waves. Based on the fingerprints of acquired spectroscopic information, the team will focus on THz wave imaging, especially on functional imaging for biomedical applications.

THz Wave Microscope: A team will develop a $\mathrm{THz}$ wave microscope to perform $\mathrm{THz}$ microspectroscopy for biomedical sensing and imaging at the cellular level. Specifically, the team will demonstrate an electro-optic $\mathrm{THz}$ wave microscope (patent pending) with a spatial resolution of $1 / 100 \lambda$ (wavelength). This non-contact and nondestructive imaging technique maintains the unique feature of providing spectroscopic information in a frequency range from 0.1 to $10 \mathrm{THz}$.

Plasma Wave Electronics for Terahertz Emission and Detection: A team will fabricate new nonlinear plasma wave electronic devices, and achieve efficient and intense terahertz emissions from solid-state devices with twodimensional electron gas. This project includes the design and fabrication of high mobility heterostructure fieldeffect transistors, capable of resonant terahertz emission and suitable for the generation of terahertz at higher harmonics, as well as new detector, for GaN-based samples, with a much higher two-dimensional electron gas density.

THz Biochip Sensor: A team will develop biochip technology for the detection of monomolecular layers and ultra low concentrations of biomolecules. One of the approaches to biochip technology will be based on metallic grating couplers. Due to plasmon surface resonance, these gratings will increase the interaction between molecular layers on its surface and the terahertz wave by several orders of magnitude. The enhanced sensitivity is expected to allow sensing and fingerprinting of monomolecular layers. THz biosensors will allow non-invasive biomonitoring and control.

Mathematical Modeling and Computer Science Infrastructure: To fully develop the applications of $\mathrm{THz}$ spectroscopy, T-ray microscope, and biochip sensors, the Center will require a sophisticated mathematical and computer science infrastructure. The process of generating and detecting the $\mathrm{THz}$ waves generates massive spectroscopic data sets. Mathematical modeling techniques such as inverse problems, functional data analysis, data mining, and machine learning are at the heart of this knowledge extraction problem.

\section{Major THz Research Efforts at Rensselaer}

THz research is currently conducted at Rensselaer in three major areas: electronics, data transfer and networking systems, and spectroscopy and imaging, as shown in Figure 1.

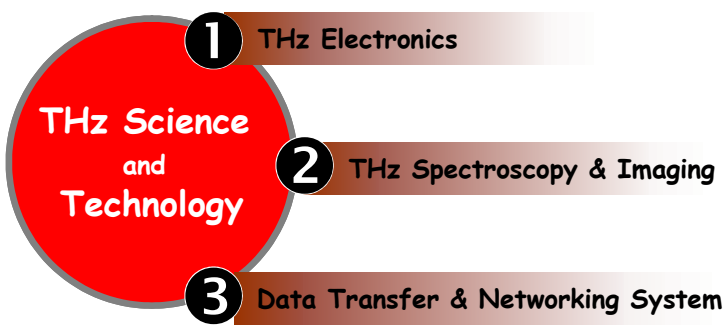

Fig. 1. Major program research themes.

\section{A. THz Electronics}

The upper frequency limit of transistors operating in conventional regimes is limited by the transit time of carriers under the gate for a field effect transistor (FET) or across the base and collector depletion region (bipolar junction transistor). The scaling of feature size has pushed device parameters to the point that transistor operation at a few hundred $\mathrm{GHz}$ is feasible. However, device feature sizes have now approached values at which fundamental physics limitations lead to diminishing returns on any further investment in scaling, and alternative device concepts have been proposed. Here, we describe several concepts that are being explored at Rensselaer. 


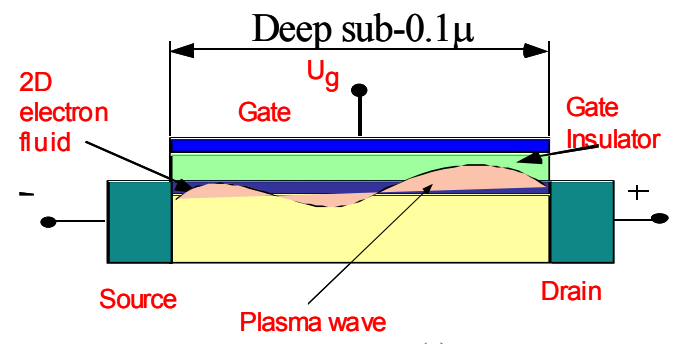

(a)

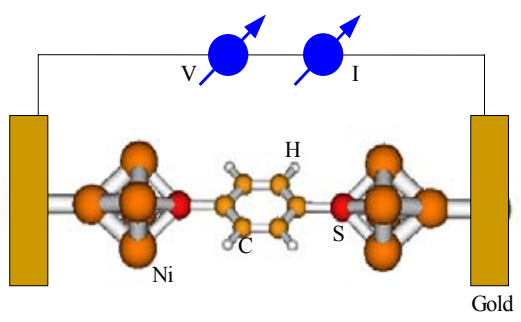

(b)

Fig. 2. (a) A THz plasma wave device. (b) Schematic of the model molecular spintronics device. Benzene-1,4-dithiolate is sandwiched between two magnetic Ni layers, followed by a nonmagnetic gold contact from which electrons are injected. The alignment of spins in the magnetic layers at the two opposite sides of the molecule controls the current passing through the circuit.

THz plasma wave electronic devices $\mathrm{THz}$ plasma-wave electronics rely on the excitation of plasma waves in the electronic fluid in high electron mobility transistor (HEMT) channels for the resonant tunable detection and excitation of $\mathrm{THz}$ radiation [1]. The waves propagating in this 2D-electron fluid are plasma waves, and their dispersion law is similar to that for shallow water waves (or sound waves), shown in Figure 2(a). This analogy has profound consequences for understanding the physics of 2D electrons in the Ballistic FET. Recently, we demonstrated operation of discrete THz resonant HEMT detectors at $2.5 \mathrm{THz}$ and $600 \mathrm{GHz}$ [2]. We will continue this effort to develop THz plasma-wave electronic technology. Specifically, we will develop THz plasma-wave electronics detector technology that uses arrays of sub-micron FETs.

Molecular spintronics Another approach to terascale electronics is to create devices down to nanoscale dimensions. Our particular interest is in the area of molecular electronics. The controlled transport of electrons/holes through a single molecule attached to electrodes forms the basis of molecular electronics. While the idea of utilizing electron spin in addition to charge to control electrical conduction in electronic circuits (spintronics) [3, 4] was discovered some time ago, almost all experiments and theory on molecular wires [5-12] have thus far considered only the charge of conduction electrons (Figure 2(b)). Spintronics devices are attractive for many applications ranging from memory storage and magnetic sensors to quantum computing devices in which electron spins would represent a quantum bit of information.

\section{B. THz Spectroscopy and Imaging}

THz electromagnetic radiation between the infrared and microwave bands is often referred to as the "terahertz gap". Compared to medical applications in the microwave, optical and x-ray frequencies, THz radiation methods are not well developed [13]. Perhaps the greatest potential for THz spectroscopy lies in biomedicine [14]. In many cases, $\mathrm{THz}$ radiation can potentially create sharper, safer, more informative images at a lower cost than conventional Xrays. THz radiation can provide spectroscopic information about the chemical composition as well as the shape and location of the targets it passes through or scatters from. THz rays are non-ionizing, which means they are noncarcinogenic, and they do not need heavy lead shielding. They can be focused, creating much sharper pictures. These unique characteristics of $\mathrm{THz}$ sensing and imaging technologies may improve the accuracy and reliability of cancer detection. Figure 3 shows such an example recently achieved.

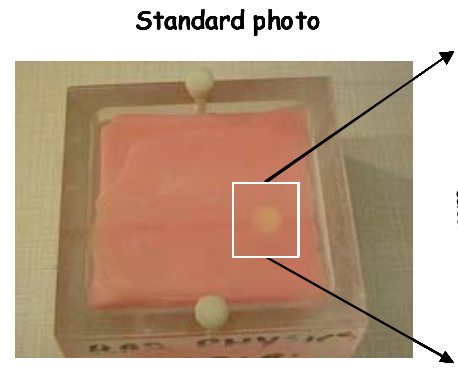

A tumor

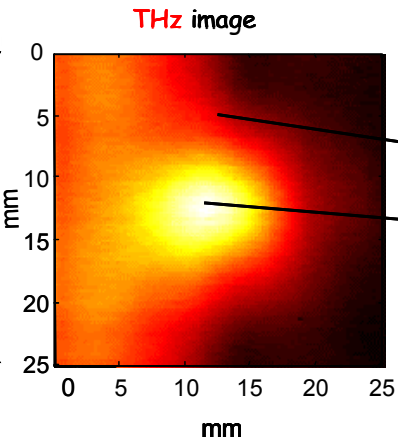

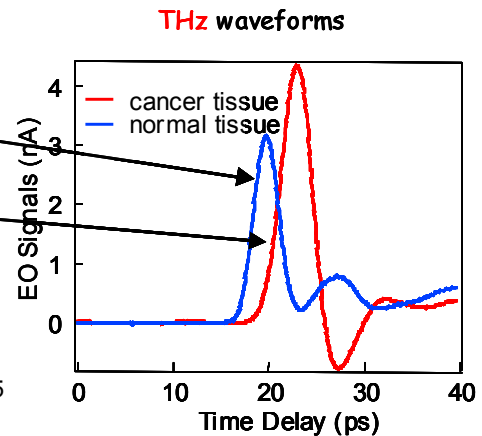

Fig. 3. THz imaging of a phantom tumor, including the spectroscopic information. 
$\mathrm{THz}$ tomography, including computed tomography ( $\mathrm{THz} \mathrm{CT}$ ), diffractive tomography, and tomography with binary lenses, is a new tomographic imaging modality that allows pulsed terahertz radiation to probe the dielectric properties of three-dimensional (3D) structures [15]. It provides sectional images of objects in an analogous manner to conventional computed tomography techniques such as X-ray CT. THz CT systems directly measure the transmitted amplitude and phase of broadband $\mathrm{THz}$ pulses at multiple projection angles. This allows a wealth of information to be extracted from the target object, including both its 3D structure and its frequency-dependent farinfrared optical properties. Our current research includes: a) Long distance/large target $\mathrm{THz}$ imaging, b) Reconstruction algorithms for THz tomography, c) 3D identification of materials, and d) Fresnel binary lens for THz imaging [16-18].

\section{C. THz Data Transfer and Networking Systems}

The need for ever-larger information technology systems is being driven by massive growth in Internet traffic, ever-increasing repositories of information and electronic services, and new computationally intensive challenges at a pace greatly outpacing Moore's law. Currently, we are addressing basic research issues in $\mathrm{THz}$ and electrical data transport, switching, and processing that enable the massive scaling required by these systems. These research challenges span materials, devices, systems, and architecture technologies and will require truly interdisciplinary research. Here we describe selective projects in this research area.

Terahertz photonics Terahertz photonics addresses signal processing at $\mathrm{THz}$ frequencies. The concept is to use few-cycle THz pulses as information units (bits). While electronic resonances limit the speed of conventional electronics (Figure 4), THz photonics can overcome this barrier and allow signal processing at $\mathrm{THz}$ speeds. Of further advantage is the fact that $\mathrm{THz}$ pulses can propagate on metallic transmission lines, which allows hybridfree on-chip integration. The most important future application area for $\mathrm{THz}$ photonics is ultrafast data communications, where $\mathrm{THz}$ photonic devices may bridge the gap between the speed of fibers and complementary metal oxide semiconductor (CMOS) technology. Recently, we have demonstrated a $\mathrm{THz}$ modulator device that makes use of intersubband excitation dynamics [19, 20]. Future research will address the main challenges for the development of photonic $\mathrm{THz}$ logics.

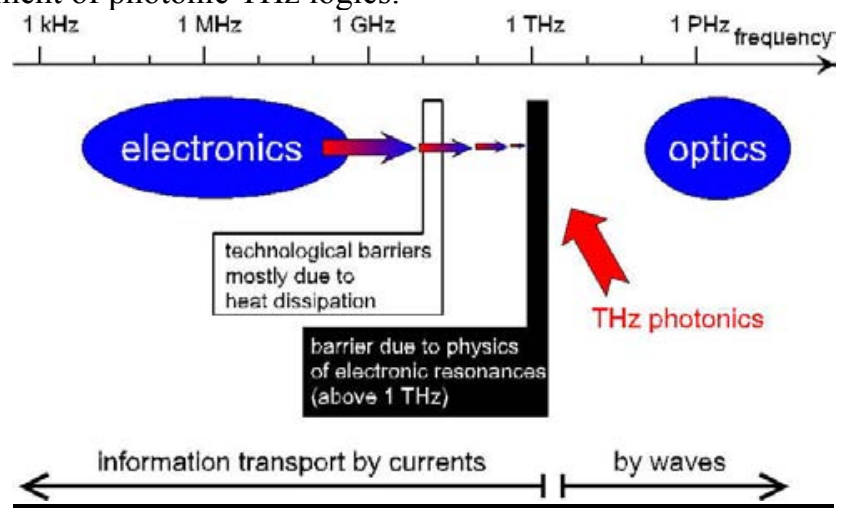

Fig. 4. Electronic resonances of semiconductors set a final barrier to all improvements of conventional devices. The resonances divide the electromagnetic spectrum into two regions. In the low- frequency region information processing can be performed conventionally by charge transport. In the high-frequency region, photonic concepts have to be applied.
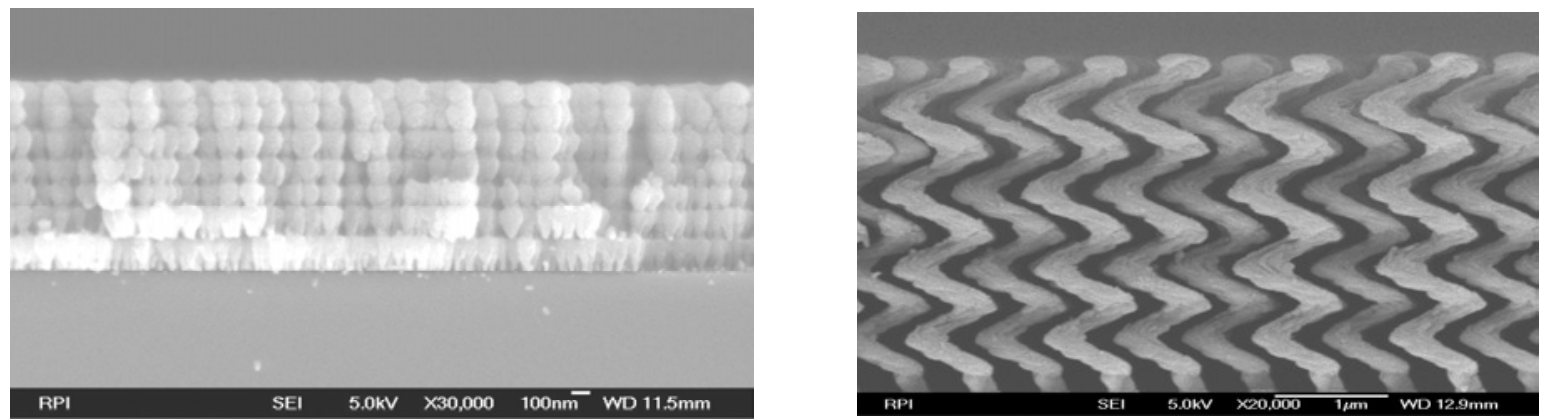

Fig. 5. SEM (scanning electron microscopy) cross-section of a photonic bandgap structures of stacked Si balls (left, scale bar $100 \mathrm{~nm}$ ) and isolated regular array of Si nanosprings (right, scale bar $1 \mu \mathrm{m}$ ) made by using the oblique angle deposition technique. 
Off-chip optical communications As computer data rates become larger and wider, it becomes increasingly difficult to support these bandwidths across MCM (multichip module) dimensions. Tightly integrated $\mathrm{MCM} /$ Optoelectronic transceivers that support high bandwidth transmission across larger distances offer a solution. In this project, we will develop materials and waveguide designs that support off-chip optical communications on a ceramic MCM. Project focus areas include waveguides on MCMs (process compatible) and chip-to-waveguide interfaces (including optical vias). We are also exploring the use of novel 3D photonic bandgap structures for possible loss-less switches and guides in photonic systems. An example is shown in Fig. 5. It is created by a single deposition step that uses an oblique angle deposition technique [21-26]. This structure cannot be fabricated using conventional lithographic techniques.

Broadband networking systems The tremendous explosion of bandwidth in the core of the Internet has generally stopped short of end-users due to the well-known "last-mile" problem: The economic infeasibility of installing high bandwidth conduits to homes and businesses. "Fiberless" or Free Space Optical (FSO) networks [2729] can effectively complement unlicensed-spectrum RF-based wireless local area networks (WLAN) technologies [30-32] to resolve this problem. Although optical networking has become popular in the wired networking world, free space optics (FSO) is still a niche technology used to provide only selected point-to-point links. Several fundamental aspects of free space terrestrial optical communication are yet to be explored. Using trans-receiver pairs that achieve $100 \mathrm{Mbps}$ with 1-10 $\mathrm{mW}$ power, we propose dense integration of thousands of trans-receivers into 2dimensional and 3-dimensional spatial structures. The 2-d array would provide extremely high aggregate bandwidth (100 Gbps and more) over 1-2 km. The 3-d spherical structures (optical antennas) would be combined with LOS auto-discovery and LOS auto-tracking techniques to instantly discover and track LOS if it exists. This work is being performed in collaboration with networking entrepreneurs such as Martin Schoffstall to conduct field trials of a mix of our prototype low-cost FSO-based systems, off-the-shelf 802.11x (wireless international standards) systems, IP routing, and $802.1 \mathrm{~d}$ bridging protocols.

\section{Detection of Defects in Space Shuttle Foam with THz Imaging Technology}

In this section, we present a recent example of detecting large size defects using THz imaging. Since April 4, 2003, we have been working with Lockheed Martin and NASA Langley Research Center to apply terahertz wave tomographic imaging technology for detecting defects in space shuttle insulating foam samples. Four samples with fabricated defects have been tested. All defects were successfully detected in the small foam samples. In the large foam samples, as shown in Figure 6, Terahertz (THz) imaging identified 49 out of 57 fabricated defects in a Protuberance Air Load (PAL) Ramp Panel with an area of 2 feet by 2 feet and a thickness ranging from 2 to 9 inches.

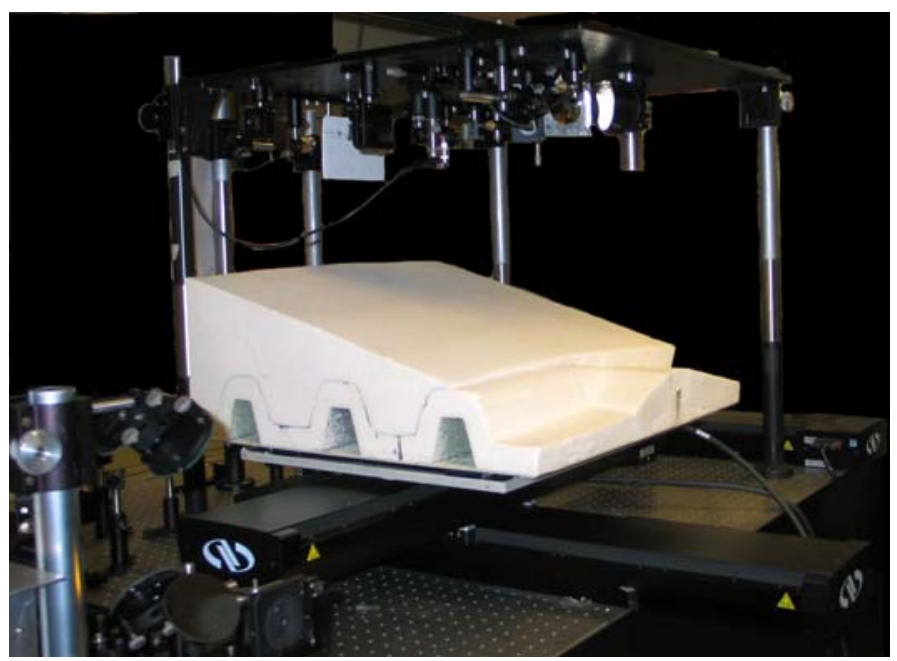

Fig. 6. Photograph of Pal Ramp Panel, SOFI (Sprayed-On Foam Insulation) foam sample and the T-ray imaging setup. 
T-ray imaging has been selected by NASA as one of four potential modalities to detect defects in foam insulation. The four modalities include: T-ray imaging, back-scattered X-ray imaging, microwave imaging and laser shearography. Rensselaer research team believes that this is a perfect research and education opportunity to apply $\mathrm{THz}$ wave sensing and imaging technology for real-world application. Figures 7 and 8 show Rensselaer's research team with Dr. Eric Madaras (NASA Langley Research Center), and two graduate students (Ms. Hua Zhong and Mr. $\mathrm{Xu} \mathrm{Xie)}$ performing measurement.

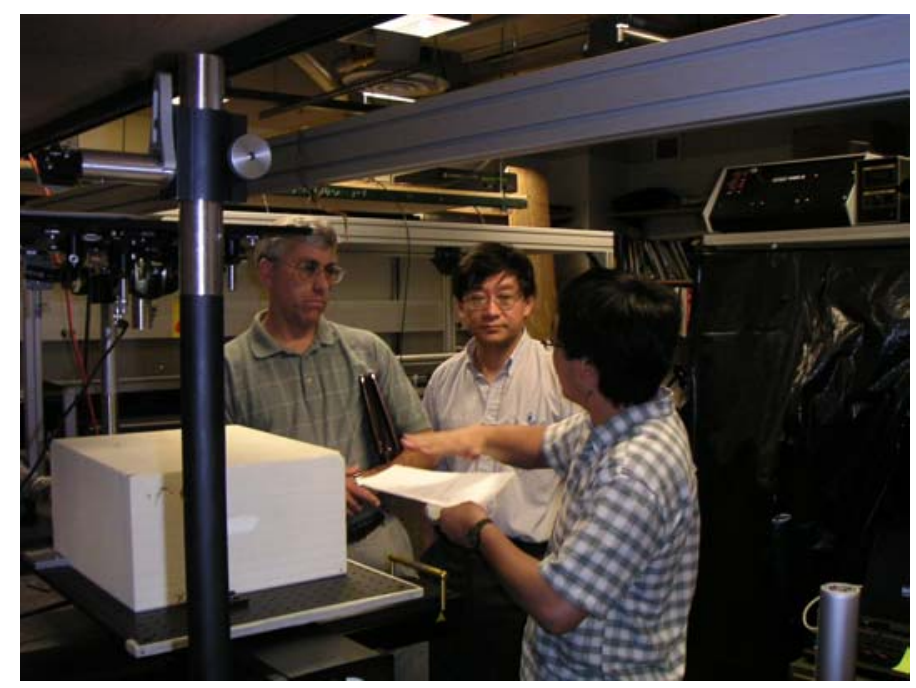

Fig. 7. NASA scientist, Dr. Madaras (left), and Rensselaer's Researchers, Prof. Zhang (center) and Dr. Xu (right), examining the THz imaging technology for the detection of defects in the space shuttle foams.

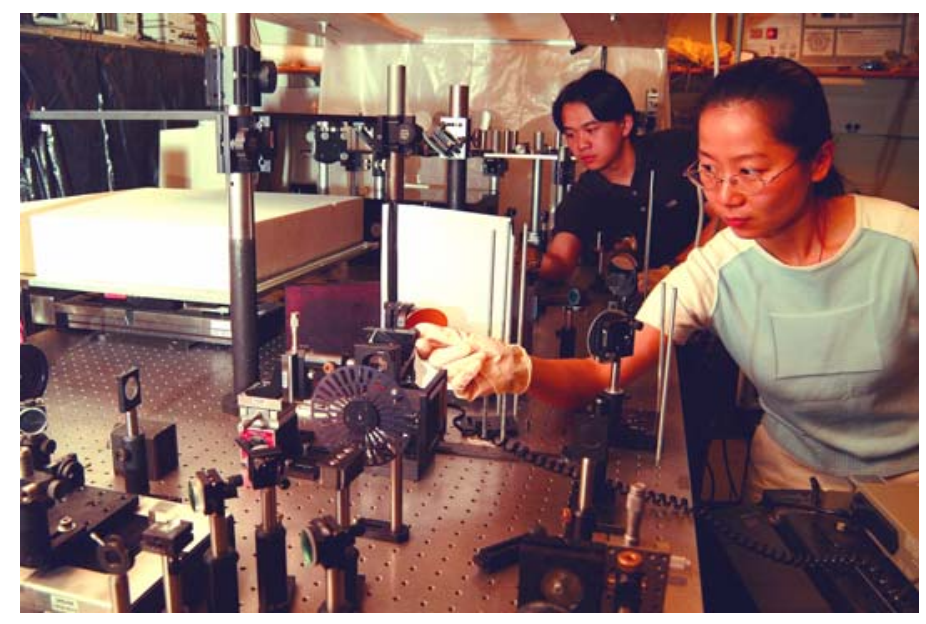

Fig. 8. Ph.D. students, Xu Xie (left) and Hua Zhong tested one of the foam samples.

The Sprayed-On Foam Insulation (SOFI) used to coat the shuttle fuel tank has low attenuation below $1 \mathrm{THz}$. This allows T-rays to penetrate through several inches of foam to identify defects buried inside. Compared to conventional technologies, which provide only intensity information of different pixels, T-ray time-domain imaging records an entire waveform of a THz pulse for each pixel. As a result, T-ray imaging provides multidimensional information of scanned objects. For example, when reflected $\mathrm{THz}$ pulses are recorded from layers, T-ray imaging visualizes the defects located in the different layers (Figure 9).

The Pal Ramp Panel sample (Figure 6) has three stringers and two flanges at its aluminum base. A layer of conethane separates the two foam layers that have been sprayed on the base. This sample includes two types of defects which may potentially occur in the foam on the fuel tank: voids (or air bubbles) and debonds (or delams), which are separations between layers of foam or between a foam layer and the aluminum base, and range 
from one-quarter-inch to two inches. The defects were placed as deep as nine inches and as shallow as a quarterinch from the top of the sample. The defects are not only located at the aluminum base and inner layers of the foam, but also at the bottom or side of the stringers and flanges. T-ray imaging located 49 of 57 defects, imaging from top and side views. The T-rays were unable to reach three defects.

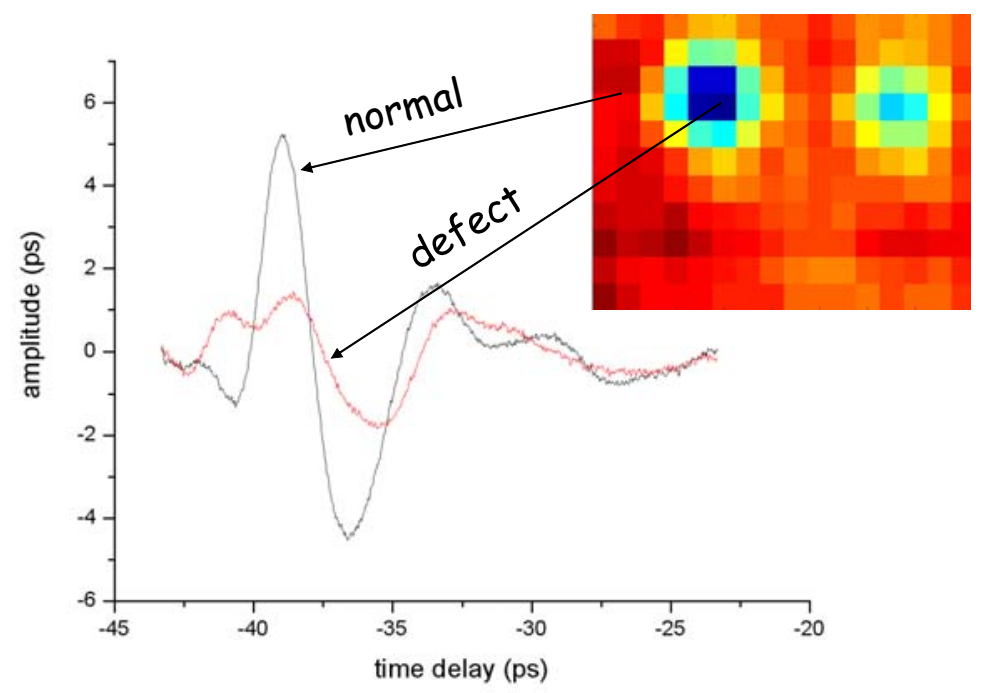

Fig. 9. THz pulses reflected from different sample foam layers. THz temporal waveform distortion from a defect is shown. The color pattern at upper right corner shows the reconstructed local image with two void defects with $1 \mathrm{~cm}$ resolution.

The T-ray imaging system was set up to scan samples from the top side of the sample. THz radiation is generated from a large aperture GaAs antenna illuminated by a femtosecond laser, and then focused onto the sample using a four-inch focal length parabolic mirror. The $\mathrm{THz}$ radiation reflected by a sample is collected by a mirror beside the parabolic mirror, and then focused onto the detection crystal by another parabolic mirror. The $\mathrm{THz}$ waveform is then recorded using a $<110>\mathrm{ZnTe}$ crystal.

When a THz pulse passes through a defect (either a void or a big gap debond), the timing and amplitude of its waveform may be modulated because the $\mathrm{THz}$ pulse passes through less foam at the defect point than at its neighboring normal area. In this case, comparing the peak timing and amplitude of the $\mathrm{THz}$ pulse for different pixels identifies the defect. When the $\mathrm{THz}$ waveforms are reflected and recorded from the foam layers, the defects located at various depths may be recorded.

Since the foam and base are not uniform for the entire sample, the variation of peak timing and amplitude of $\mathrm{THz}$ pulse along the entire foam sample may be even larger than the difference between the defect and its neighboring area. As a result, the defects will be buried in the larger background variation. To solve this problem, a median filter is applied to the image and subtracted from the original to single out the abnormal point.

Certain defects, such as small gap debonds (which do not cause a sufficient change of timing and amplitude of the $\mathrm{THz}$ pulse to provide image contrast), may generate a $\mathrm{THz}$ temporal waveform distortion. To emphasize the waveform distortion, we calculate the standard deviation or cross correlation of the $\mathrm{THz}$ waveform at each pixel with a standard waveform. In the cross correlation or standard deviation map, the defects are clearly defined among the regular points.

Figures 10(a) and 4(b) map detected sample foam defects (near the substrate and near the center layer of the foam). There are a total of 57 manufactured sample foam defects, including 25 voids and 32 debonds. Compared to the debonds, the voids show higher contrast in the THz image. T-rays can visualize all 25 voids measuring 1.5 to 0.25 inches. T-rays were able to detect 24 of the 32 debonds; three were not be reached by the THz waves. Debonds larger than one inch clearly appear in the THz image, and those measuring less than 0.5 inch are difficult to detect. 


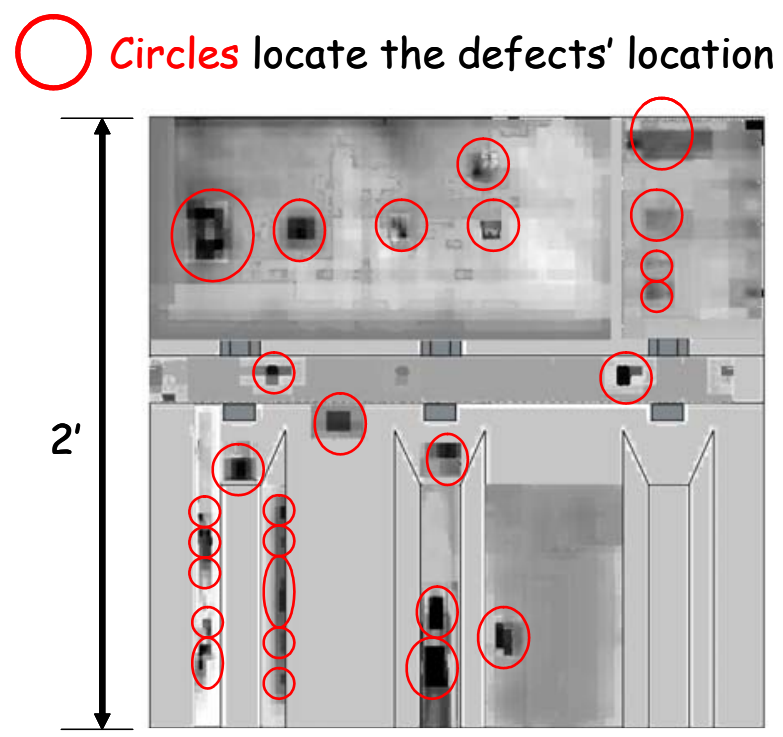

(a)

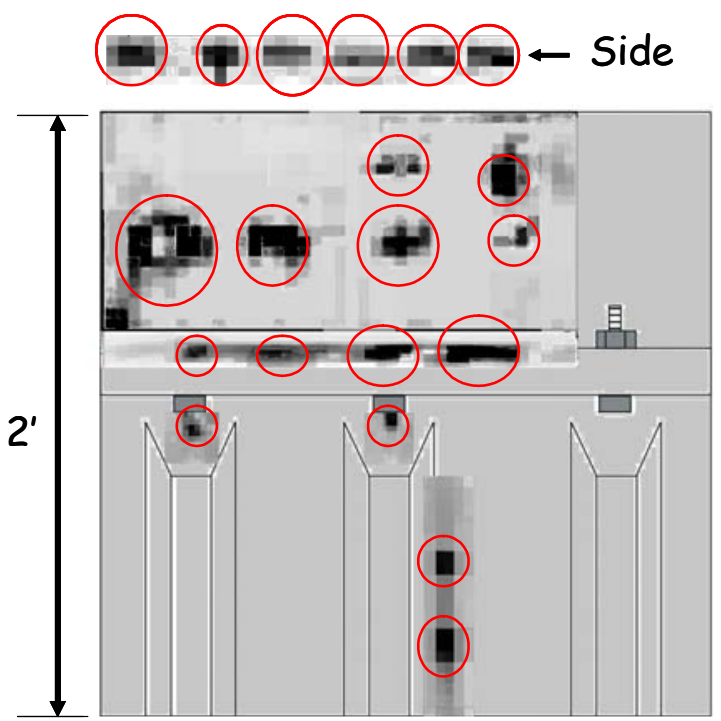

(b)

Fig. 10. T-ray image of defects in sample foam. (a) Images of defects created by using a THz pulse reflected from the base. (b) Images of defects created by using a THz pulse reflected from the inner layer. The defect spots are indicated on the construction map of the Pal Ramp Panel in Fig. 6.

\section{Studio-Based Education and Soft Skills Training}

In addition to the strong commitment to research, there is an equal dedication to education, including an expanded and enhanced doctoral program. Rensselaer has won numerous awards for its revolutionary studio-based approach to undergraduate education, including the first Pew Charitable Trust Award for the Renewal of Undergraduate Education and the first Boeing Outstanding Educator Award. The Institute is now adapting and extending the pedagogical principles successfully implemented under this program to doctoral education for students working at the leading edge of science and technology. The goal is to implement an integrated studio program that will enhance the depth and breadth of our students' understanding while at the same time equipping them with the tools that will be needed for successful careers. Figure 11 shows typical faculty and student interactions in a technology-enriched learning environment.

\section{Technology \& Multimedia}

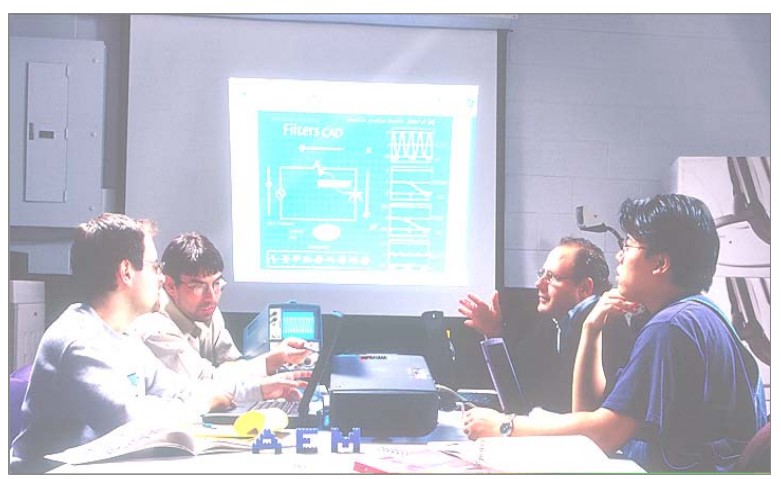

\section{Interactive Learning}

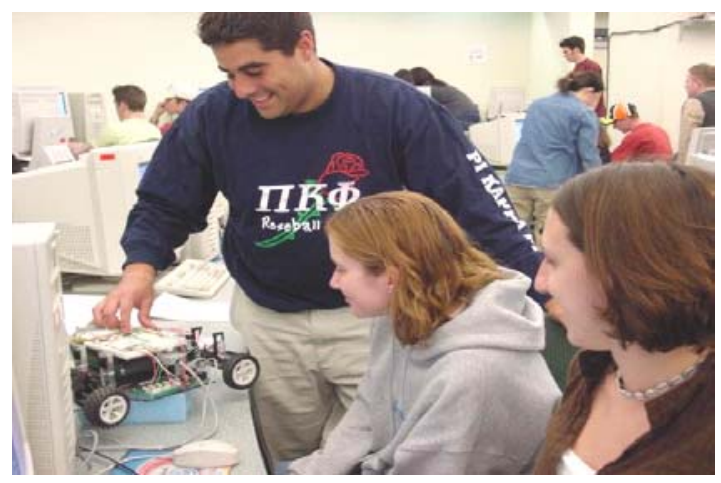

\section{Studio Learning and Teaching Environment}

Fig. 11. Studio based education and soft skills training at Rensselaer Polytechnic Institute.

The IGERT project will integrate our programs in THz materials, devices, radiation, imaging, spectroscopy and computer algorithms to create an environment in which students learn from each other, from on-campus researchers, and from our industrial partners while forming a global network of collaborators and contacts. The curriculum will be highly interdisciplinary. PhD students in THz science and technology will need fundamentals, breadth, depth, and 
experimental research experience in a wide range of subjects, including quantum mechanics, non-linear optics, chemistry, materials, devices, optoelectronics, networking systems, and wireless communication. Rensselaer's strength in these multiple fields provides a unique opportunity to develop a crosscutting interdisciplinary graduate program in the studio mode. Students will build THz-related research skills by choosing fundamental courses in optics and THz science and technology, as well as optional courses in related fields designed to bring breadth to the curriculum. In addition, there will be short courses and seminars delivered by faculty members and by U.S. and International collaborators in the $\mathrm{THz}$ research program.

In addition to an innovative curriculum, we will create an infrastructure that nurtures organization, teamwork, communication, mentoring, leadership, and ethics in our students. The IGERT students are required to attend workshops designed by the Rensselaer Archer Center for Student Leadership Development team. This one credit "Professional Development" and "Life Skills" series requires students to be exposed to specific leadership theories and skills, including ethical decision making, developing vision, motivation techniques, communications, teamwork, multiculturalism, and tools to succeed in a diverse organizational culture.

As part of our effort to foster diversity, we will develop a close working relationship with historically black universities and colleges (HBCU). For example, in order to initiate research, HBCU faculty will collaborate with the Rensselaer THz program. A pipeline will allow HBCU students to conduct research at Rensselaer as well as at their home campus and will encourage them to consider the doctoral program in $\mathrm{THz}$ science and technology upon graduation.

\section{Conclusion}

Since 1992, Rensselaer's terahertz research team members have received grants from both government agencies, private foundations and industries. Examples are the National Science Foundation, Army Research Office, Army Research Laboratory, Air Force Office of Scientific Research, Department of Energy, Defense Advanced Research Projects Agency, W.M. Keck Foundation, Research Corporation, IMRA America Incorporated, Molecular OptoElectronic Corporation, 3D Digital Corp., and Zomega Technology Corporation.

Using these grants, the terahertz center's labs are equipped with the most advanced photonic and opto-electronic instrumentation for generating, measuring and recording picosecond and femtosecond terahertz radiation waves. The Center will take the lead to use these labs and facilities to create studio-based new courses in major THz research areas for students. Rensselaer's Center for Terahertz Research stands at the forefront of terahertz technology, we expect $\mathrm{THz}$ science and technology to become one of the most promising research areas for transformational imaging in the $21^{\text {st }}$ century, and we educate and train first rate scientists for this challenging transformation.

\section{Acknowledgement}

The authors thank NSF, ARO, Keck Foundation, DARPA, Rensselaer and many other generous supports that allow the research, education and training in Terahertz science and technology possible at Rensselaer.

\section{References}

1. "Detection, mixing, and frequency multiplication of terahertz radiation by two dimensional electronic fluid", M. I. Dyakonov and M. S. Shur, IEEE Transactions on Electron Devices, Vol. 43, No. 3, pp. 380-387, March (1996).

2. "Resonant detection of sub-terahertz radiation by plasma waves in the submicron field effect transistor", W. Knap, Y. Deng, S. Rumyantsev, J.-Q. Lu, M. S. Shur, C. A. Saylor, and L. C. Brunel, Appl. Phys. Lett., Vol. 80, No 18, pp. 3433-3435, 6 May (2002).

3. "Magnetoelectronics", G. A. Prinz, Science 282, 1660 (1998).

4. "Interfacial charge-spin coupling: Injection and detection of spin magnetization in metals", M. Johnson and R.H. Silsbee, Phys. Rev. Lett. 55, 1270 (1985).

5. "Electronics using hybrid-molecular and mono-molecular devices", C. Joachim, J. K. Gimzewski, and A. Aviram, Nature (London) 408, 541 (2000).

6. "Conductance of a molecular junction", M. A. Reed, C. Zhou, C. J. Muller, T. P. Burgin, and J. M. Tour, Science 278, 252 (1997).

7. "Driving current through single organic molecules", J. Reichert, R. Ochs, D. Beckmann, H. B. Weber, M. Mayor, and H. v. Löhneysen, Phys. Rev. Lett. 88, 176804 (2002). 
8. "Electronically configurable molecular-based logic gates", C. P. Collier, E. W. Wong, M. Belohradsky, F. M. Raymo, J. F. Stoddart, P. J. Kuekes, R. S. Williams, and J. R. Heath, Science 285, 391 (1999).

9. "Room-temperature negative differential resistance in nanoscale molecular junctions", J. Chen, W. Wang, M. A. Reed, A. M. Rawlett, D. W. Price, and J. M. Tour, App. Phys. Lett. 77, 1224 (2000).

10. "Molecular random access memory cell", M. A. Reed, J. Chen, A. M. Rawlett, D. W. Price, and J. M. Tour, App. Phys. Lett. 78, 3735 (2001).

11. "First-principles calculation of transport properties of a molecular device", M. Di Ventra, S. T. Pantelides, and N. D. Lang, Phys. Rev. Lett. 84, 979 (2000).

12. "Molecular spintronics: Spin-dependent electron transport in molecular wires", E. Emberley and G. Kirczenow, cond-mat/0201344.

13. "Materials for terahertz science and technology", B. Ferguson and X.-C. Zhang, Review Article, Nature Materials, 1, 26 (2002).

14. "Terahertz biosensing technology: Frontiers and progress", Abdellah Menikh, Robert MacColl, Carmen A. Mannella, and Xi-Cheng Zhang, Highlight Article, ChemPhysChem, 3, 655-658 (2002).

15. "T-ray computed tomography", Bradley Ferguson, Shaohong Wang, Doug Gray, Derek Abbott, and X.-C. Zhang, Optics Letters, 27, 1312 (2002).

16. "Characterization of T-ray binary lenses", S. Wang, T. Yuan, E. D. Walsby, R. J. Blaikie, S. M. Durbin, D. R. S. Cumming, J. Xu, and X.-C. Zhang, Optics Letters, 27, 1183 (2002).

17. "Terahertz wave tomography with a Fresnel lens", S. Wang and X.-C. Zhang, submitted to Optics Letters (2002).

18. "Characterization of T-ray binary lenses", S. Wang, T. Yuan, E.D. Walsby, R.J. Blaikie, S.M. Durbin, D.R.S. Cumming, J. Xu, and X.-C. Zhang, Opt. Lett. 27, 1183 (2002).

19. "Sampling a THz dipole transition with sub-cycle time-resolution", R. Kersting, R. Bratschitsch , G. Strasser , K. Unterrainer, and J.N. Heyman, Opt. Lett. 25, 272 (2000).

20. "Terahertz phase modulator", R. Kersting, G. Strasser, and K. Unterrainer, Electr. Lett. 36, 1156 (2000).

21. "Square spiral phonic crystals: Robust architecture for microfabrication of materials with large threedimensional photonic band gap", Ovidiu Toader and Sajeev John, Phys. Rev. E 66, 016610 (2002).

22. "Optical properties of photonic crystals" by Kazuaki Sakoda, Springer Series in Optical Sciences (2001).

23. "2D and 3D photonic crystals built with VLSI tools", S. Lin et al., MRS Bulletin, 26, 627, August (2001).

24. Photonic crystals: Molding the flow of light, J.D. Joannopoulos, R.D. Meade, and J.N. Winn (Princeton University Press, 1995).

25. "Novel nano-column and nano-flower arrays by glancing angle deposition”, Y.-P. Zhao, D.-X. Ye, G.-C. Wang, and T.-M. Lu, Nano Letters 2, 351 (2002).

26. "Fabrication of Si nanocolumns and Si square spirals on self-assembled monolayers colloid substrates", Y.-P. Zhao, D.-X. Ye, Pei-I Wang, G.-C. Wang, and T.-M. Lu, International Journal of Nanoscience, 1, 87 (2002).

27. "Wireless infrared communications", J.M. Kahn and J.R. Barry, Proc. of the IEEE, Vol. 85, pp. 265-298 (1997).

28. "Wireless optical transmission of fast ethernet, FDDI, ATM, and ESCON protocol data using the TerraLink laser communication system", I. I. Kim et al., SPIE Opt. Eng., Vol. 37, No. 12, pp. 3143-3155 (1998).

29. "A broadband wireless access network based on mesh-connected free-space optical links", A.S. Acampora et al., IEEE Personal Communications, Vol. 6, pp. 62 -65 (1999).

30. "Fiberless optical communication links for isolated communities", J.C.A. Chaimowicz et al., Second International Conference on Rural Telecommunications, pp. 202-206 (1990).

31. "Design aspects of hybrid RF/free space optical wireless networks", T. ElBatt et al., IEEE Emerging Technologies Symp. on Broadband Communications for the Internet Era, Symp. Digest, pp. 157-161 (2001).

32. "A millimeter wave broadband wireless access technology demonstrator for the next generation internet network reach extension”, H. Izadpanah, IEEE Communications Magazine, Vol. 39, pp. 140-145 (2001). 